\title{
Curvature tensor in tangette bundles of semi-riemannian manifold
}

\author{
Cigdem Inci Kuzu ${ }^{1}$ and Nejmi Cengiz ${ }^{2}$ \\ ${ }^{1}$ Department of Mathematics and Science Education, Ibrahim Çeçen University of Ağri, Ağri, Turkey \\ ${ }^{2}$ Department of Mathematics, Atatürk University, Erzurum, Turkey
}

Received: 17 April 2018, Accepted: 18 May 2018

Published online: 11 August 2018.

\begin{abstract}
In the conducted study, some theorems have been written by calculating $\tilde{R}_{k i j}^{h}$ coefficient of ${ }^{H} R$ curvature tensor and $\tilde{S}_{i j}^{h}$ coefficient of ${ }^{H} S$ torsion tensor according to affine connection in tangente bundles of Semi-Riemannian manifold. Besides, ${ }^{H} R_{i j}$ Ricci tensor has been examined and ${ }^{H} R_{i j}$ coefficient has been calculated. Finally, $S={ }^{H} g{ }^{I H}{ }_{R}$ scalar curvature has been examined and
\end{abstract} some theorems have been associated with this.

Keywords: Semi-Riemannian manifold, curvature tensor, torsion tensor, Ricci tensor, scalar curvature.

\section{Introduction}

In this study, $\tilde{S}_{i j}^{h}$ coefficients of ${ }^{H} R$ Curvature Tensor and $\tilde{R}_{k i j}^{h}$ and ${ }^{H} S$ Torsion Tensor were calculated according to the affine connection in the tangent bundle of the Semi-Riemannianian manifold. Defined on $M$ manifold.

(i) $g(X, Y)=g(Y, X), \forall X, Y \in \mathfrak{I}_{0}^{1}(M)$,(balancing).

(ii) $g(X, X) \geq 0, \forall X \in \mathfrak{I}_{0}^{1}(M)$ ve $g(X, X)=0 \Leftrightarrow X=0$, (Positive definition).

$(0,2)$-typed $g$ tensor field fulfilling the conditions is called as Riemannian metric or metric tensor. In this case, $\left(M_{n}, g\right)$ pair is called as Riemannian manifold.

Let $M n$ be an $n$-dimensional differentiable manifold of and $C^{\infty}$ class and $T^{1} q(M n)$ the tensor bundle over $M n$ of tensor of type $(1, q)$. If $x i$ are local coordinates in a neighborhood $U$ of point $x \in M n$, then a tensor $t$ at $x$ which is an element of $T^{1} q(M n)$ is expressible in the form $\left(x i, t j i_{1} \cdots i q\right)$, where $t j i_{1} \ldots i q$ are components of $t$ with respect to the natural frame. It may be considered $\left(x^{i}, t_{i 1}^{j} \ldots i_{q}\right)=\left(x^{i}, x^{\bar{i}}\right)=\left(x^{J}\right), i=l, \ldots, n, \bar{i}=n+1, \ldots, n\left(1+n^{q}\right), I=1, \ldots, n(l+n q)$ as local coordinates in a neighborhood $\pi^{-1}$ is the natural projection $T q^{1}(M n)$ onto $\left.M n\right)$.

Let then $M n$ be a Riemannian manifold with non-degenerate metric $g$ whose components in a coordinate neighborhood $U$ are $g j i$ and denote by $\Gamma$ hji the Christoffel symbols which are formed withgji.

We indicate by $\mathfrak{I}_{s}^{r}\left(M_{n}\right)$ the module over $F(M n)\left(F(M n)\right.$ is the ring of $C^{\infty}$ functions in $M n$ all tensor fields of $C^{\infty}$ class and of type $(r, s)$ in $M n$. Let $X \in \mathfrak{I}_{0}^{1}\left(M_{n}\right)$ and $w \in \mathfrak{I}_{q}^{1}\left(M_{n}\right)$. Then ${ }^{C} X \in \mathfrak{I}_{0}^{1}\left(T_{q}^{1}\left(M_{n}\right)\right)$ (complete lift) ${ }^{H} X \in \mathfrak{I}_{0}^{1}\left(T_{q}^{1}\left(M_{n}\right)\right)$ (horizontal lift) and ${ }^{V} w \in \mathfrak{I}_{0}^{1}\left(T_{q}^{1}\left(M_{n}\right)\right)$ (vertical lift) have, respectively, components. [1,2,3]

For the curvature tensor of connection $\forall X, Y, Z \in \mathfrak{I}_{0}^{1}\left(M_{n}\right)$

$$
R(X, Y, Z)=\nabla_{X} \nabla_{Y} Z-\nabla_{Y} \nabla_{X} Z-\nabla_{[X, Y]} Z,
$$

it is defined as above $[4,5,6,7]$. Instead of $R(X, Y, Z), R(X, Y) Z$ can also be used.

$$
R(X, Y, Z)=-R(Y, X, Z),
$$


is understood from (1). It is easy to see that $R$ fulfills linearity condition in terms of $X, Y$ and $Z$ variables. However, if $R(X, Y, Z) \in \mathfrak{I}_{0}^{1}\left(M_{n}\right), R \in \mathfrak{I}_{3}^{1}\left(M_{n}\right)$.

In accompany with (1) if it is considered that $X=\partial_{i}, Y=\partial_{j}, Z=\partial_{k}$, the coordinates of $R$ on the natural framework are expressed as following [8],

$$
R_{i j k}^{s}=\partial_{i} \Gamma_{j k}^{s}-\partial_{j} \Gamma_{i k}^{s}+\Gamma_{i m}^{s} \Gamma_{j k}^{m}-\Gamma_{j m}^{s} \Gamma_{i k}^{m} .
$$

The torsion tensor of the connection is defined as,

$$
2 S(X, Y)=\nabla_{X} Y-\nabla_{Y} X-[X, Y] \forall X, Y \in \mathfrak{I}_{0}^{1}\left(M_{n}\right)
$$

In this expression, if it is considered that $X=\partial_{i}, Y=\partial_{j}$, the coordinates of $S$ on the natural framework are,

$$
S_{i j}^{k}=\frac{1}{2}\left(\Gamma_{i j}^{k}-\Gamma_{j i}^{k}\right)
$$

It is seen that $S_{i j}^{k}=-S_{j i}^{k}$. Using the (4) equation, coefficients of $\tilde{S}_{i j}^{h}$ were calculated. Ricci tensor is the tensor defined by utilizing $R$ curvature tensor,

$$
R_{i j}=R_{k i j}^{k}
$$

If curvature tensor formula is used,

$$
R_{i j}=R_{k i j}^{k}=\partial_{k} \Gamma_{i j}^{k}-\partial_{i} \Gamma_{k j}^{k}+\Gamma_{k l}^{k} \Gamma_{i j}^{l}-\Gamma_{k i}^{l} \Gamma_{l j}^{k}
$$

That means, if $\Gamma_{k s}^{s}=\partial_{k} \ln e, R_{i j}=R_{k i j}^{k}$ Ricci tensor is symmetrical. This means that Ricci tensor can be indicated as $R_{i j}=R_{j i}$. In tension free spaces, if the equation $R_{[i j k]}^{s}=\frac{1}{3}\left(R_{i j k}^{s}+R_{j k i}^{s}+R_{k i j}^{s}\right)=0$, is used,

$$
R_{r s k}^{k}=R_{r s}-R_{s r}
$$

is obtained [4]. $M_{n}$ is a $n$-dimensioned Riemannian manifold among $C^{\infty}$ class, let $g_{i j}$ metric be regular, symmetrical and let the connection be Levi-Civita connection. On $M_{n}$, if the $s$ index on $R_{i j k}^{s}$ curvature tensor is moved down to the place after $k,(0,4)$-typed tensor indicated below is obtained.

$$
R_{i j k t}=g_{s t} R_{i j k}^{s} \Leftrightarrow R(X, Y, Z, W)=g(R(X, Y) Z, W)
$$

$R_{i j}=R_{s i j}^{s}=g^{t s} R_{t i j s}=g^{t s} R_{i t s j}$ tensor is called as Ricci tensor [9].

Full contraction operation is conducted with $g^{i j}$ tensor and Ricci tensor and

$$
R=g^{i j} R_{i j}
$$

The $R$ curvature here is called as scaler curvature. If the pseudo-Riemannian metric indicated as $g$ on $M_{n}$ is defined as $d s^{2}=g_{j i} d x^{j} d x^{i}$, the pseudo-Riemannian metric indicated as ${ }^{H} g$ on $T\left(M_{n}\right)$ is $2 g_{j i} \delta y^{j} d x^{i}$ [10]. Here, $\delta y^{j}=d y^{j}+\tilde{\Gamma}_{l k}^{j} y^{l} d x^{k}$, moreover, both $\Gamma_{l k}^{j}$ and $M_{n} \nabla$ affine connection coefficients and the covariant components of ${ }^{H} g$ metric on tangent cluster are $[11,12]$;

$$
{ }^{H} g^{I H}=\left(\begin{array}{cc}
0 & g^{i h} \\
g^{i h} & -\left(\Gamma_{j}^{h} g^{j i}+\Gamma_{s}^{i} g^{s h}\right)
\end{array}\right)
$$

Using ${ }^{H} R_{i j}$ coefficients and ${ }^{H} g^{I H}=\left(\begin{array}{c}0 \\ g^{i h}-\left(\Gamma_{j}^{h} g^{j i}+\Gamma_{s}^{i} g^{s h}\right.\end{array}\right)$ equation, the coefficients of $S={ }^{H} g^{I H H} R_{I J}$ were calculated.

\section{$2{ }^{H} R$ Curvature and ${ }^{H} S$ Torsion tensors on the tangent bundle of semi-Riemannian manifold according to affine connection}

${ }^{H} \Gamma_{J K}^{I}$ is the symbol of Cristoffel defined with ${ }^{H} g$. Using ${ }^{H} \Gamma_{J K}^{I}=\frac{1}{2}{ }^{H} g^{I S}\left(\partial_{J}{ }^{H} g_{S K}+\partial_{K}{ }^{H} g_{J S}-\partial_{S}{ }^{H} g_{J K}\right)$ formula, each coefficient of ${ }^{H} \Gamma_{J K}^{I}$ was calculated [11]. We get, 


$$
\begin{aligned}
& { }^{H} \Gamma_{j k}^{i}=\Gamma_{j k}^{i}+\frac{1}{2} g^{i s} \nabla_{s} g_{j k},{ }^{H} \Gamma_{j \bar{k}}^{i}=0,{ }^{H} \Gamma_{\bar{j} k}^{i}=0,{ }^{H} \Gamma_{j k}^{i}=0,{ }^{H} \Gamma_{j k}^{\bar{i}}=0,{ }^{H} \Gamma_{\bar{j} k}^{\bar{i}}=\Gamma_{j k}^{i}-\frac{1}{2} g^{i s} \nabla_{j} g_{s k},{ }^{H} \Gamma_{j \bar{k}}^{\bar{i}}=\Gamma_{j k}^{i}-\frac{1}{2} g^{i s} \nabla_{k} g_{j s}, \\
& { }^{H} \Gamma_{j k}^{\bar{i}}=y^{l} \partial_{l} \Gamma_{j k}^{i}-y^{l}\left(\nabla_{l} g^{i s}\right) g_{i s} \Gamma_{j k}^{i},-\frac{1}{2} g^{i s} y^{l}\left(\partial_{j}\left(\nabla_{l} g_{s k}\right)+\partial_{k}\left(\nabla_{l} g_{j s}\right)-\partial_{s}\left(\nabla_{l} g_{j k}\right)\right),+\frac{1}{2} y^{l}\left(\nabla_{l} g^{i s}\right)\left(\nabla_{s} g_{j k}\right)+\frac{1}{2} y^{l} \partial_{l} g^{i s} \nabla_{s} g_{j k} .
\end{aligned}
$$

Theorem 1. $\nabla, M_{n}$ is an affine connection in $\nabla, M_{n}$ manifold, the necessary and sufficient condition for ${ }^{C} \nabla$ full lift and ${ }^{H} \nabla$ horizontal lift to be equal is that $\nabla$ is a metric connection.

Using $\tilde{R}_{K J I}^{H}=\partial_{K}{ }^{H} \Gamma_{J I}^{H}-\partial_{J}{ }^{H} \Gamma_{K I}^{H}+{ }^{H} \Gamma_{K T}^{H}{ }^{H} \Gamma_{J I}^{T}-{ }^{H} \Gamma_{J I}^{H H} \Gamma_{K I}^{T}$ formula and each of the coefficients of ${ }^{H} \Gamma_{J K}^{I}$, the $\tilde{R}_{k j i}^{h}$ coefficients of ${ }^{H} R$ curvature tensor were calculated. It is found that

$$
\begin{aligned}
& \tilde{R}_{k j i}^{h}=\partial_{k}{ }^{H} \Gamma_{j i}^{h}-\partial_{j}{ }^{H} \Gamma_{k i}^{h}+{ }^{H} \Gamma_{k T}^{h H} \Gamma_{j i}^{T}-{ }^{H} \Gamma_{j T}^{h H} \Gamma_{k i}^{T} \\
& =\partial_{k}{ }^{H} \Gamma_{j i}^{h}-\partial_{j}{ }^{H} \Gamma_{k i}^{h}+\left({ }^{H} \Gamma_{k t}^{h H} \Gamma_{j i}^{t}+{ }^{H} \Gamma_{k \bar{t}}^{h H} \Gamma_{j i}^{\bar{t}}\right)-\left({ }^{H} \Gamma_{j t}^{h H} \Gamma_{k i}^{t}+{ }^{H} \Gamma_{j \bar{t}}^{h H} \Gamma_{k i}^{\bar{t}}\right) \\
& =\partial_{k}\left(\Gamma_{j i}^{h}+\frac{1}{2} g^{h s} \nabla_{s} g_{j i}\right)-\partial_{j}\left(\Gamma_{k i}^{h}+\frac{1}{2} g^{h s} \nabla_{s} g_{k i}\right)+\left[\left(\Gamma_{k t}^{h}+\frac{1}{2} g^{h s} \nabla_{s} g_{k t}\right) \cdot\left(\Gamma_{j i}^{t}+\frac{1}{2} g^{t s} \nabla_{s} g_{j i}\right)\right] \\
& -\left[\left(\Gamma_{j t}^{h}+\frac{1}{2} g^{h s} \nabla_{s} g_{j t}\right) \cdot\left(\Gamma_{k i}^{t}+\frac{1}{2} g^{t s} \nabla_{s} g_{k i}\right)\right] \\
& \left.=\partial_{k} \Gamma_{j i}^{h}+\frac{1}{2} \partial_{k}\left(g^{h s} \nabla_{s} g_{j i}\right)-\partial_{j} \Gamma_{k i}^{h}-\frac{1}{2} \partial_{j}\left(g^{h s} \nabla_{s} g_{k i}\right)+\Gamma_{k t}^{h} \Gamma_{j i}^{t}+\frac{1}{2} \Gamma_{k t}^{h} g^{t s} \nabla_{s} g_{j i}+\frac{1}{2} \Gamma_{j i}^{t} g^{h s} \nabla_{s} g_{k t}\right) \\
& +\frac{1}{4} g^{h s} g^{t s} \nabla_{s} g_{k t} \nabla_{s} g_{j i}-\Gamma_{j t}^{h} \Gamma_{k i}^{t}-\frac{1}{2} \Gamma_{j t}^{h} g^{t s} \nabla_{s} g_{k i}-\frac{1}{2} \Gamma_{k i}^{t}{ }^{h s} \nabla_{s} g_{j t}-\frac{1}{4} g^{h s} g^{t s} \nabla_{g} g_{j t} \nabla_{s} g_{k i} \\
& =R_{k j i}^{h}+\frac{1}{2}\left(\partial_{k} g^{h s} \nabla_{s} g_{j i}\right)-\partial_{j}\left(g^{h s} \nabla_{s} g_{k i}\right)+\frac{1}{2} g^{t s}\left(\Gamma_{k t}^{h} \nabla_{s} g_{j i}-\Gamma_{j t}^{h} \nabla_{s} g_{k i}\right) \\
& +\frac{1}{2} g^{h s}\left(\Gamma_{j i}^{t} \nabla_{s} g_{g t}-\Gamma_{k i}^{t} \nabla_{s} g_{j t}\right)+\frac{1}{4} g^{h s} g^{t s}\left(\nabla_{s} g_{k t} \nabla_{s} g_{j i}-\nabla_{s} g_{j t}+\nabla_{s} g_{k i}\right) \\
& \tilde{R}_{k j i}^{\bar{h}}=R_{k j i}^{h} \\
& \tilde{R}_{k j \bar{i}}^{\bar{h}}=R_{k j i}^{h}-\frac{1}{2}\left(\partial_{k}\left(g^{h s} \nabla_{j} g_{i s}\right)-\partial_{j}\left(g^{h s} \nabla_{k} g_{i s}\right)\right)-\frac{1}{2} g^{t s}\left(\Gamma_{k t}^{h} \nabla_{i} g_{j s}-\Gamma_{j t}^{h} \nabla_{i} g_{k s}\right) \\
& -\frac{1}{2} g^{h s}\left(\Gamma_{j i}^{t} \nabla_{t} g_{k s}-\Gamma_{k i}^{t} \nabla_{t} g_{j s}\right)+\frac{1}{4} g^{h s} g^{t s}\left(\nabla_{t} g_{k s} \nabla_{i} g_{j s}-\nabla_{t} g_{j s} \nabla_{i} g_{k s}\right) \\
& \tilde{R}_{k \bar{h} i}^{\bar{h}}=\partial_{k}\left(\Gamma_{j i}^{h}-\frac{1}{2} g^{h s} \nabla_{j} g_{s i}\right)-\left(\partial_{j} \Gamma_{k i}^{h}-\nabla_{j} g^{h s}\right) g_{h s} \Gamma_{k i}^{h} \\
& -\frac{1}{2} g^{h s} y^{\ell}\left(\partial_{k}\left(\nabla_{\ell} g_{s i}\right) \partial_{i}\left(\nabla_{\ell} g_{k s}\right)-\partial_{s}\left(\nabla_{\ell} g_{k i}\right)\right) \\
& \left.+\frac{1}{2} y^{\ell}\left(\nabla_{\ell} g^{h s}\right)\left(\nabla_{s} g_{k i}\right)+\frac{1}{2} y^{\ell} \partial_{\ell} g^{h s} \nabla_{s} g_{k i}\right] \\
& \tilde{R}_{\bar{k} j i}^{\bar{h}}=\left(\partial _ { k } \left(y^{l} \partial_{l} \Gamma_{j i}^{h}-y^{l}\left(\nabla_{l} g^{h s}\right) g_{h s} \Gamma_{j i}^{h}-\frac{1}{2} g^{h s} y^{l}\left(\partial_{j}\left(\nabla_{l} g_{s i}\right)+\left(\partial_{i}\left(\nabla_{l} g_{j s}\right)-\left(\partial_{s}\left(\nabla_{l} g_{j i}\right)\right.\right.\right.\right.\right. \\
& \left.\left.+\frac{1}{2} y^{l}\left(\nabla_{l} g^{h s}\right)\left(\nabla_{s} g_{j i}\right)+\frac{1}{2} y^{l} \partial_{l} g^{h s} \nabla_{s} g_{j i}\right)\right)-\left(\partial_{j}\left(\Gamma_{k i}^{h}-\frac{1}{2} g^{h s} \nabla_{k} g_{s i}\right)\right) \\
& +\left(\left(\Gamma_{k t}^{h}-\frac{1}{2} g^{h s} \nabla_{k} g_{s t}\right)\left(\Gamma_{j i}^{t}-\frac{1}{2} g^{t s} \nabla_{j} g_{s i}\right)-\left(\left(\Gamma_{j t}^{h}-\frac{1}{2} g^{h s} \nabla_{t} g_{s j}\right)\left(\Gamma_{k i}^{t}-\frac{1}{2} g^{t s} \nabla_{i} g_{k s}\right)\right)\right.
\end{aligned}
$$

and

$$
\tilde{R}_{\bar{k} j i}^{h}=\tilde{R}_{\bar{k} \bar{j} i}^{h}=\tilde{R}_{\bar{k} \overline{j i}}^{h}=\tilde{R}_{k \bar{j} i}^{h}=\tilde{R}_{k j \bar{i}}^{h}=\tilde{R}_{\bar{k} \bar{j} i}^{h}=\tilde{R}_{k \bar{j} i}^{h}=\tilde{R}_{k \overline{j i}}^{\bar{h}}=\tilde{R}_{\bar{k} \bar{j} i}^{\bar{h}}=\tilde{R}_{\bar{k} \overline{j i}}^{\bar{h}}=\tilde{R}_{\bar{k} j \bar{i}}^{\bar{h}}=0 .
$$

Result. Let $\left(M_{n}, g\right)$ be semi-Riemannian manifold. According to metric connection, $\tilde{R}_{i j k}^{h}$ coefficients of $R$ tensor are as 
following on $(\nabla g=0)$ tangent bundle,

$$
\begin{gathered}
\tilde{R}_{k j i}^{h}=R_{k j i}^{h}, \tilde{R}_{k j i}^{\bar{h}}=R_{k j i}^{h} \tilde{R}_{k j \bar{i}}^{\bar{h}}=R_{k j i}^{h}, \tilde{R}_{\bar{k} j i}^{\bar{h}}=R_{k j i}^{h}, \\
\tilde{R}_{k \bar{j} i}^{\bar{h}}=\partial_{k} \Gamma_{j i}^{h}-\partial_{j} \Gamma_{k i}^{h} g_{h s} \Gamma_{k i}^{h},
\end{gathered}
$$

and the others are zero.

The torsion tensor of the connection is,

$$
S_{i j}^{k}=\frac{1}{2}\left(\Gamma_{i j}^{k}-\Gamma_{j i}^{k}\right)
$$

Using (5) equation, $\tilde{S}_{i j}^{h}$ coefficients were calculated. They are

$$
\begin{aligned}
\tilde{S}_{j i}^{h}= & \frac{1}{2}\left({ }^{H} \Gamma_{j i}^{h}-{ }^{H} \Gamma_{i j}^{h}\right) \\
= & \frac{1}{2}\left(\Gamma_{j i}^{h}+\frac{1}{2} g^{h s} \nabla_{s} g_{j i}-\Gamma_{i j}^{h}-\frac{1}{2} g^{h s} \nabla_{s} g_{i j}\right) \\
= & \frac{1}{2}\left(\Gamma_{j i}^{h}-\Gamma_{i j}^{h}+\frac{1}{2} g^{h s} \nabla_{s} g_{j i}-\frac{1}{2} g^{h s} \nabla_{s} g_{i j}\right) \\
= & S_{j i}^{h}+\frac{1}{4} g^{h} \nabla_{s}\left(g_{j i}-g_{i j}\right) \\
& \tilde{S}_{\bar{h}}^{\bar{h}}=S_{j i}^{h}, \\
& \tilde{S}_{j i}^{h}=S_{j i}^{h}, \\
& \tilde{S}_{j i}^{\bar{h}}=S_{j i}^{h} y^{\ell}\left(\partial_{\ell}-\left(\nabla_{\ell} g^{h s}\right) g_{h s},\right) \\
& \tilde{S}_{j i}^{h}=\tilde{S}_{j \bar{i}}^{h}=\tilde{S}_{j i}^{h}=\tilde{S}_{j i}^{\bar{h}}=0 .
\end{aligned}
$$

Theorem 2. Torsion-free space which has metric connection on $\left(M_{n}, g\right)$ semi-Riemannian manifold tangent bundle is $S^{h}=0$.

\section{Analysis of ${ }^{H} R_{i j}$ Ricci tensor on tangent bundle}

Using,

$$
R_{r s k}^{k}=R_{r s}-R_{s r}
$$

and $\tilde{R}_{k j i}^{h}$ coefficients, ${ }^{H} R_{i j}$ coefficients were calculated. They are found as

$$
\begin{aligned}
{ }^{H} R_{j i} & =R_{K j i}^{K}=R_{k j i}^{k}+R_{\bar{k} j i}^{\bar{k}}=R_{k j i}^{k}+\frac{1}{2}\left(\partial_{k}\left(g^{k s} \nabla_{s} g_{j i}\right)-\left(\partial_{j}\left(g^{k s} \nabla_{s} g_{k i}\right)+\frac{1}{2} g^{t s}\left(\Gamma_{k t}^{k} \nabla_{s} g_{j i}-\Gamma_{j t}^{k} \nabla_{s} g_{k i}\right)\right.\right. \\
& +\frac{1}{2} g^{k s}\left(\Gamma_{j i}^{t} \nabla_{s} g_{k t}-\Gamma_{k i}^{t} \nabla_{s} g_{j t}\right)+\frac{1}{4} g^{k s} g^{t s}\left(\nabla_{s} g_{k t} \nabla_{s} g_{j i}-\nabla_{s} g_{j t} \nabla_{s} g_{k i}\right) \\
& +\left(\partial _ { k } \left(y^{l} \partial_{l} \Gamma_{j i}^{k}-y^{l}\left(\nabla_{l} g^{k s}\right) g_{k s} \Gamma_{j i}^{k}-\frac{1}{2} g^{k s} y^{l}\left(\partial_{j}\left(\nabla_{l} g_{s i}\right)+\left(\partial_{i}\left(\nabla_{l} g_{j s}\right)-\left(\partial_{s}\left(\nabla_{l} g_{j i}\right)\right.\right.\right.\right.\right. \\
& \left.\left.+\frac{1}{2} y^{l}\left(\nabla_{l} g^{k s}\right)\left(\nabla_{s} g_{j i}\right)+\frac{1}{2} y^{l} \partial_{l} g^{k s} \nabla_{s} g_{j i}\right)\right)-\left(\partial_{j}\left(\Gamma_{k i}^{k}-\frac{1}{2} g^{k s} \nabla_{k} g_{s i}\right)\right) \\
& +\left(\Gamma_{k t}^{k}-\frac{1}{2} g^{k s} \nabla_{k} g_{s t}\right)\left(\Gamma_{j i}^{t}-\frac{1}{2} g^{t s} \nabla_{j} g_{s i}\right)-\left(\left(\Gamma_{j t}^{k}-\frac{1}{2} g^{k s} \nabla_{t} g_{s j}\right)\left(\Gamma_{k i}^{t}-\frac{1}{2} g^{t s} \nabla_{i} g_{k s}\right)\right)
\end{aligned}
$$




$$
\begin{aligned}
& { }^{H} R_{\overline{j i}}=R_{K \overline{j i}}^{K}=R_{k \bar{k} i}^{k}+R_{\bar{k} \bar{j} i}^{\bar{k}}=0+0=0 \\
& { }^{H} R_{j \bar{i}}=R_{K j \bar{i}}^{K}=R_{k j i}^{k}+R_{\bar{k} j \bar{i}}^{\bar{k}}=0+0=0 \\
& { }^{H} R_{\overline{j i}}=R_{K \overline{j i}}^{K}=R_{k \bar{j} \bar{i}}^{k}+R_{\bar{k} \bar{k} \bar{k}}^{\bar{k}}=0+0=0 .
\end{aligned}
$$

When $\nabla g=0$,

$$
\begin{aligned}
& { }^{H} R_{j i}=R_{k j i}^{k}+\partial_{k}\left(y^{l} \partial_{l} \Gamma_{j i}^{k}\right)-\partial_{j} \Gamma_{k i}^{k}+\Gamma_{k t}^{k} \Gamma_{j i}^{t}-\Gamma_{t j}^{k} \Gamma_{k i}^{t}, \\
& { }^{H} R_{\overline{j i}}={ }^{H} R_{\bar{j} i}={ }^{H} R_{j \bar{i}}=0 .
\end{aligned}
$$

\section{Analysis of $S={ }^{H} g^{I H H} R_{I J}$ scaler curvature on tangent bundle}

Using ${ }^{H} R_{i j}$ coefficients and ${ }^{H} g^{I H}=\left(\begin{array}{c}0 \\ g^{i h}-\left(\Gamma_{j}^{h} g^{g i}+\Gamma_{s}^{i} g^{s h}\right.\end{array}\right)$ equation, the coefficients of $S={ }^{H} g^{I H H} R_{I J}$ were calculated. They are found as,

$$
\begin{aligned}
{ }^{H} g^{i j H} R_{i j} & =0 .\left(R_{k j i}^{k}+\frac{1}{2}\left(\partial_{k}\left(g^{k s} \nabla_{s} g_{j i}\right)-\left(\partial_{j}\left(g^{k s} \nabla_{s} g_{k i}\right)+\frac{1}{2} g^{t s}\left(\Gamma_{k t}^{k} \nabla_{s} g_{j i}-\Gamma_{j t}^{k} \nabla_{s} g_{k i}\right)\right.\right.\right. \\
& +\frac{1}{2} g^{k s}\left(\Gamma_{j i}^{t} \nabla_{s} g_{k t}-\Gamma_{k i}^{t} \nabla_{s} g_{j t}\right)+\frac{1}{4} g^{k s} g^{t s}\left(\nabla_{s} g_{k t} \nabla_{s} g_{j i}-\nabla_{s} g_{j t} \nabla_{s} g_{k i}\right) \\
& +\left(\partial _ { k } \left(y^{l} \partial_{l} \Gamma_{j i}^{k}-y^{l}\left(\nabla_{l} g^{k s}\right) g_{k s} \Gamma_{j i}^{k}-\frac{1}{2} g^{k s} y^{l}\left(\partial_{j}\left(\nabla_{l} g_{s i}\right)\right.\right.\right. \\
& +\left(\partial_{i}\left(\nabla_{l} g_{j s}\right)-\left(\partial_{s}\left(\nabla_{l} g_{j i}\right)+\frac{1}{2} y^{l}\left(\nabla_{l} g^{k s}\right)\left(\nabla_{s} g_{j i}\right)+\frac{1}{2} y^{l} \partial_{l} g^{k s} \nabla_{s} g_{j i}\right)\right)-\left(\partial_{j}\left(\Gamma_{k i}^{k}-\frac{1}{2} g^{k s} \nabla_{k} g_{s i}\right)\right) \\
& +\left(\Gamma_{k t}^{k}-\frac{1}{2} g^{k s} \nabla_{k} g_{s t}\right)\left(\Gamma_{j i}^{t}-\frac{1}{2} g^{t s} \nabla_{j} g_{s i}\right)-\left(\left(\Gamma_{j t}^{k}-\frac{1}{2} g^{k s} \nabla_{t} g_{s j}\right)\left(\Gamma_{k i}^{t}-\frac{1}{2} g^{t s} \nabla_{i} g_{k s}\right)\right)=0 \\
{ }^{H} g^{i j H} R_{\bar{i} j} & =g^{i h} .0=0 \\
{ }^{H} g^{i \bar{j} H} R_{i \bar{j}} & =g^{i h} .0=0 \\
{ }^{H} g^{i \bar{j} H} R_{\bar{i} \bar{j}} & =-\left(\Gamma_{j}^{h} g^{j i}+\Gamma_{s}^{i} g^{s h}\right) .0=0
\end{aligned}
$$

Theorem 3. Let $\left(M_{n}, g\right)$ be semi-Riemannian manifold. ${ }^{H} R$ scaler curvature of $\left(T\left(M_{n},{ }^{H} g\right)\right)$ space is zero.

\section{Competing interests}

The authors declare that they have no competing interests.

\section{Authors' contributions}

All authors have contributed to all parts of the article. All authors read and approved the final manuscript.

\section{References}

[1] A. A. Salimov, A new method in theory of lifts of tensor fields to a tensor bundle, Russian Mathematics-New York, 38(3), 69-75, 1994.

[2] A. A. Salimov, Generalized Yano-Ako operator and the complete lift of tensor fields, Tensor, 55 (2), 142-146, 1994.

[3] A. A. Salimov, A. MaÇ§den, Complete lifts of tensor fields on a pure cross-section in the tensor bundle Tq1(Mn), Note Di Matematica 18 (1), 27-37, 1998. 
[4] P. B. Gilkey, Geometric properties of natural operators defined by the Riemannian curvature tensor, World Scientific Publishing, New Jersey, 2001.

[5] P. Gilkey, J. V. Leahy, H. Sadofsky, Riemannian manifolds whose skew-symmetric curvature operator has constant eigenvalues, Indiana University mathematics journal, 48 (2), 615-634, 1999.

[6] P. Gilkey, T. Zhang, Algebraic curvature tensors for indefinite metrics whose skew-symmetric curvature operator has constant Jordan normal form, Houston Journal of Mathematics, 76 (1), 1-18, 2002.

[7] S. Ivanov, I. Petrova, Riemannian manifold in which the skew-symmetric curvature operator has pointwise constant eigenvalues, Geometriae Dedicata 70 (3), 269-282, 1998.

[8] P. B. Gilkey, The moduli space of Type A surfaces with torsion and non-singular symmetric Ricci tensor, Journal of Geometry and Physics 110, 69-77, 2016.

[9] K. Yano, S. Kobayashi, Prolongations of tensor Fields and Connections to Tangent Bundles I -General Theory-, Journal of the Mathematical Society of Japan 18, 194-210, 1966.

[10] K. Yano, S. lshihara, Horizontal Lifts of Tensor Fields and Conneetions to Tangent Bundles, Jour. Math. and Mech., 16 (9), 1015-1030, 1967.

[11] I. E. Hirica, On some pseudo-symmetric Riemann spaces, Balkan Journal of Geometry and Its Applications 14 (2), $42-49,2009$.

[12] I. Kim, H. Park, H. Song, Ricci pseudo-symmetric real hypersurfaces in complex space forms, Nihonkai Mathematical Journal 18 (1-2): 1-9, 2007. 